\title{
High Frequency Conductivity in the Quantum Hall Regime
}

\author{
F. Hohls, * U. Zeitler, and R. J. Haug \\ Institut für Festkörperphysik, Universität Hannover, Applestrasse 2, 30167 Hannover, Germany
}

(Received 1 November 2000)

\begin{abstract}
We have measured the complex conductivity $\sigma_{x x}$ of a two-dimensional electron system in the quantum Hall regime up to frequencies of $6 \mathrm{GHz}$ at electron temperatures below $100 \mathrm{mK}$. Using both its imaginary and real part we show that $\sigma_{x x}$ can be scaled to a single function for different frequencies and several transitions between plateaus in the quantum Hall effect. Additionally, the conductivity in the variable-range hopping regime is used for a direct evaluation of the localization length $\xi$. Even for large filling factor distances $\delta \nu$ from the critical point we find $\xi \propto \delta \nu^{-\gamma}$ with a scaling exponent $\gamma=2.3$.
\end{abstract}

DOI: 10.1103/PhysRevLett.86.5124

PACS numbers: 73.43.-f, 71.23.An, 71.30.+h, 72.20.My

It is widely accepted that the understanding of the integer quantum Hall effect (QHE) is closely related to a disorder driven localization-delocalization transition occurring in two-dimensional electron systems (2DES) in high magnetic fields [1]. Many experimental and theoretical works approve the interpretation of the transition between adjacent QHE plateaus as a quantum critical phase transition. It is governed by a diverging localization length $\xi \propto\left|E-E_{c}\right|^{-\gamma}$ which scales with the distance of the energy $E$ from the critical energy $E_{c}$ in the center of a Landau band. The exponent $\gamma \approx 2.3$ is believed to be a universal quantity independent of disorder. For finite systems with effective size $L_{\text {eff }}$ theory predicts that the conductivities $\sigma_{\alpha \beta}$ follow scaling functions $\sigma_{\alpha \beta}=f_{\alpha \beta}\left[L_{\text {eff }} / \xi(E)\right]$ resulting in a finite width $\Delta E \propto L_{\mathrm{eff}}^{-1 / \gamma}$ of the transition region. The effective system size $L_{\text {eff }}$ is determined by the physical sample size, the electron temperature $T$, or the frequency $f$.

The most common test of scaling uses an analysis of the temperature or frequency dependence of the conductivity peak width in the QHE plateau transition. However, lacking an exact expression for $L_{\text {eff }}(T, f)$, this method does not allow direct access to the scaling behavior of the localization length.

An alternative approach to scaling was proposed by Polyakov and Shklovskii [2]. Using the fact that the conductivity $\sigma_{x x}$ in the QHE plateaus at low temperatures is dominated by variable-range hopping (VRH) [3] a direct access to the localization length $\xi$ can be gained from analyzing the dependence of $\sigma_{x x}$ on temperature, current, and frequency. Experimentally mainly the temperature dependence of $\sigma_{x x}$ in the VRH regime was investigated [4]. However, due to an unknown theoretical prefactor, $\xi$ could only be estimated from these experiments.

In contrast, the frequency driven variable-range hopping conductivity $\sigma_{x x}(f)$ [in the limit $\sigma_{x x}(f) \gg \sigma_{x x}(0)$ ] is given by [2]

$$
\operatorname{Re} \sigma_{x x}(\omega)=\frac{2 \pi}{3} \epsilon \epsilon_{0} \xi \omega,
$$

linearly depending on both frequency $f=\omega / 2 \pi$ and localization length $\xi$ with no unknown prefactors.

Here we report on measurements of the complex conductivity $\sigma_{x x}$ up to frequencies $f=6 \mathrm{GHz}$ at low temperatures down to below $100 \mathrm{mK}$. We will show that $\operatorname{Im}\left(\sigma_{x x}\right)$ can be scaled with a single-parameter function to $\operatorname{Re}\left(\sigma_{x x}\right)$, independent of temperature, frequency, and filling factor. Second, we will use Eq. (1) to directly measure the localization length $\xi$ deep into the variable-range hopping regime for several QHE plateau transitions. Its filling factor dependence follows a scaling behavior $\xi \propto$ $\left|\nu-\nu_{c}\right|^{-\gamma}$ with $\gamma=2.3$ up to large distances $\left|\nu-\nu_{c}\right| \geq$ 0.3 from the critical point $\nu_{c}$. In the center of the QHE plateaus $\xi$ is found to be limited by the magnetic length, the natural length scale of the quantum Hall state.

The two-dimensional electron system used in our experiments was realized in an $\mathrm{AlGaAs} / \mathrm{GaAs}$ heterostructure grown by molecular beam epitaxy and lies $75 \mathrm{~nm}$ underneath the surface. Its electron mobility and density are $\mu=35 \mathrm{~m}^{2} / \mathrm{Vs}$ and $n=3.3 \cdot 10^{15} \mathrm{~m}^{-2}$. The sample was patterned into Corbino geometry with contacts fabricated by standard $\mathrm{Ni} / \mathrm{Au} / \mathrm{Ge}$ alloy annealing. This geometry allows a direct two-point measurement of the longitudinal conductivity $\sigma_{x x}$ at high frequencies. For an ideal Corbino geometry $\sigma_{x x}$ is given by $2 \pi \sigma_{x x}=$ $G \ln \left(r_{2} / r_{1}\right) . G=I / U$ is the two-point conductance with current $I$ and voltage $U$ measured at the same contacts. The sample dimensions of our Corbino ring are $r_{2}=$ $820 \mu \mathrm{m}$ for the outer and $r_{1}=800 \mu \mathrm{m}$ for the inner radius.

The conductance measurement at high frequencies is realized by a reflection measurement setup: The sample acts as load of a high frequency coaxial line with a characteristic impedance of $Z_{0}=50 \Omega$. A load impedance $Z=$ $1 / G$ deviating from $Z_{0}$ leads to reflection of an incident wave at the load with a complex reflection coefficient $\mathcal{R}_{S}=\left(Z-Z_{0}\right) /\left(Z+Z_{0}\right)$. The total reflection coefficient $\mathcal{R}$ of the loaded line is made up of the sample reflection $\mathcal{R}_{S}$ and of properties of the line itself such as phase shifts, losses, and reflections at interconnections. A careful 
calibration of these frequency dependent contributions of the line allows the extraction of $\mathcal{R}_{S}(f)$ and therefore of the complex sample conductivity $\sigma_{x x}(f)$ from the direct accessible quantity $\mathcal{R}(f)$. The sample dimensions were chosen as a compromise between sensitivity, which is largest for a sample impedance $Z$ close to $Z_{0}=50 \Omega$, and the avoidance of size effects for too small ring widths [5].

The sample and coaxial line were fitted into a dilution refrigerator with base temperature $T_{S}<50 \mathrm{mK}$ using a multistep thermal sinking of the line. The sample is situated in the center of a superconducting solenoid capable of producing magnetic fields up to $B=15 \mathrm{~T}$. The reflection $\mathcal{R}$ of the coaxial line terminated with the sample is measured with a network analyzer with a frequency range $f=100 \mathrm{kHz}$ to $6 \mathrm{GHz}$ using power levels $P \leq-75 \mathrm{dBm}$ which were checked not to influence the measured conductivity. The analyzer was used in continuous wave mode with fixed frequency while stepping the magnetic field.

Figure 1 shows the real part $\operatorname{Re}\left(\sigma_{x x}\right)$ of the measured conductivity as a function of the filling factor $\nu=$ $n h / e B$. The larger measurement noise with rising frequency is a consequence of lower transmission of the coaxial lines and thus a stronger damped signal at high frequencies. The plot shows well pronounced Shubnikovde Haas oscillations with zero conductivity at integer filling factors and maxima near half integer $\nu$. Every peak corresponds to the transition between adjacent quantum Hall states with a critical point $\nu_{c}$ at maximum

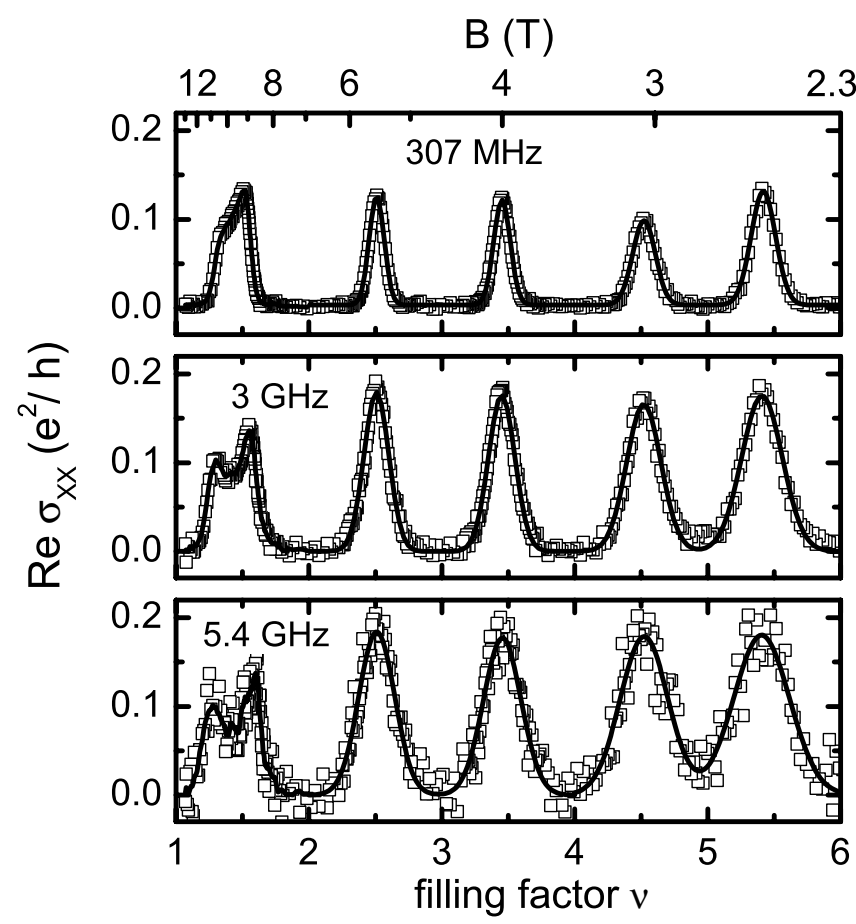

FIG. 1. Real part of the conductivity at different frequencies. The squares are the raw data points, the line is derived by Gaussian fits for $\nu>2$ and simple smoothing for $\nu<2$. conductivity. The transition $\nu=1 \rightarrow 2$ within the lowest Landau level deviates from the other transitions by form and amplitude of the peak and shows a shoulder possibly originating from the presence of an impurity band [6].

The other transitions are very symmetric and can be fitted by Gaussians. This fit is used to derive the position of the critical filling factors $\nu_{c}$ and the critical conductivities $\sigma_{c}$. For low frequencies the conductivity is lowered by a frequency dependent contact resistance present in our two-point measurement [7]. At higher frequencies this effect becomes negligible and one measures the pure bulk conductivity. For $f \geq 2 \mathrm{GHz}$ and $\nu>2$ the value $\sigma_{c} \approx$ $0.17 e^{2} / h$ is the same for all transitions and independent of frequency as expected by scaling theory, but deviates from the proposed universal value $0.5 e^{2} / h$ [8]. A possible explanation for this discrepancy was given by Ruzin et al. [9], who proposed fluctuations in the carrier density as the cause of nonuniversal critical conductivity values.

Previous experiments by Engel et al. [10] and Balaban et al. [11] were restricted to a measurement of the real part of $\sigma_{x x}$, whereas our technique gives access to both the real and imaginary part. This allows an additional test of universal frequency scaling. As an example we show the real and the imaginary part of the complex conductivity $\sigma_{x x}$ for $f=3 \mathrm{GHz}$ in Fig. 2a. $\operatorname{Im}\left(\sigma_{x x}\right)$ and $\operatorname{Re}\left(\sigma_{x x}\right)$ display a similar symmetry around the critical points, which are marked by the maxima of the conductivity. In the QHE plateau centers between two critical points $\sigma_{x x}$ tend to zero (except for $\nu=5$ where spin splitting is no longer fully resolved). Approaching the critical points both $\operatorname{Im}\left(\sigma_{x x}\right)$ and $\operatorname{Re}\left(\sigma_{x x}\right)$ start to rise to positive value. While to our knowledge there is no published theoretical prediction of the imaginary part of the hopping conductivity of interacting electrons in high magnetic fields this behavior agrees qualitatively with the theory of Efros [12] for low magnetic fields. He calculated $\operatorname{Im}\left(\sigma_{x x}\right) \propto \ln \left(\omega_{\mathrm{ph}} / \omega\right) \operatorname{Re}\left(\sigma_{x x}\right)$ with $\omega_{\mathrm{ph}}$ the characteristic phonon frequency, which gives a linear dependence between $\operatorname{Im}\left(\sigma_{x x}\right)$ and $\operatorname{Re}\left(\sigma_{x x}\right)$ at fixed frequency.

When moving closer towards the critical point, $\operatorname{Im}\left(\sigma_{x x}\right)$ starts dropping back to a value near zero, whereas $\sigma_{x x}$ continues increasing up to $\sigma_{x x}^{\max }=0.17 e^{2} / h$ for the sample investigated. This agrees with the expectation for a quasimetallic behavior of a $2 \mathrm{DES}$ at the critical point. The crossover between the variable-range hopping regime and the metallic regime occurs at a conductivity $\operatorname{Re}\left(\sigma_{x x}\right) \approx$ $0.4 \sigma_{x x}^{\max }$, independently of frequency $(f \geq 1 \mathrm{GHz})$ and temperature.

The extra information gained from the imaginary part allows a new test of the applicability of scaling theory. For comparison between theory and experiment one can use the relation $\delta E=E-E_{c} \propto \delta \nu=\nu-\nu_{c}$ in the filling factor range $0 \leq \delta \nu \lesssim 0.3$. Here, the error is less than $10 \%$ in a Gaussian model of the density of states. The theory predicts a complex conductivity following a scaling function $\sigma_{x x}(f, \delta \nu)=g_{x x}\left[L_{f} / \xi(\delta \nu)\right]$ [13], where 

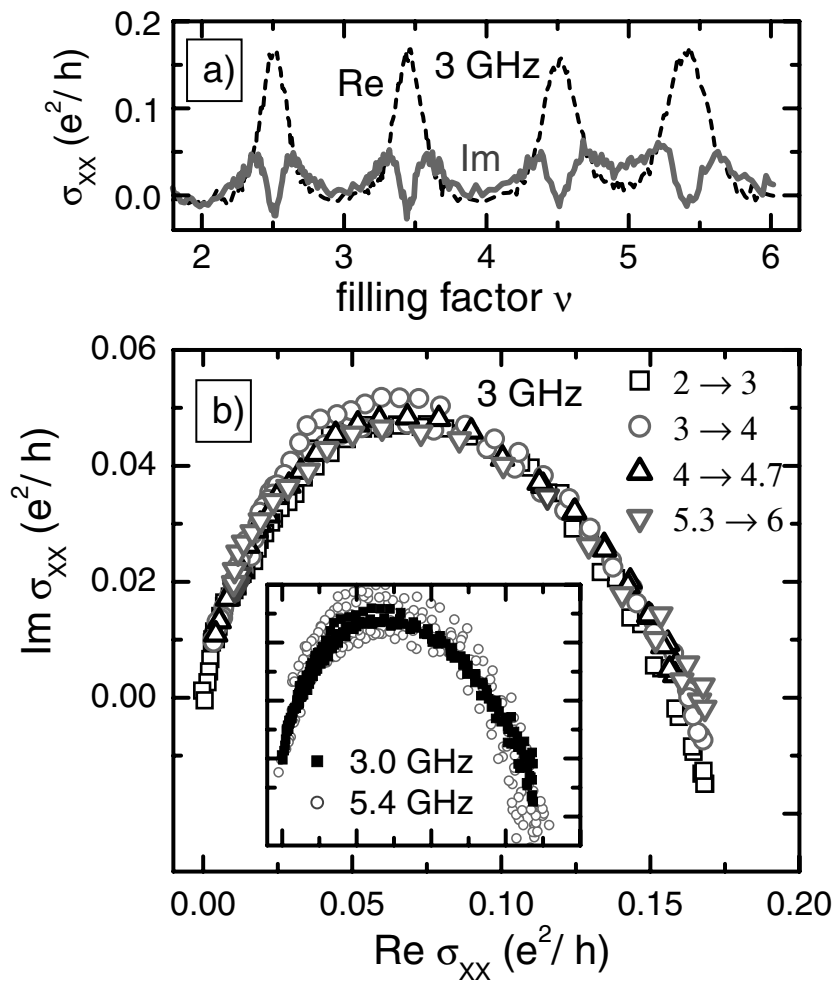

FIG. 2. (a) Real (broken line) and imaginary (full line) part of $\sigma_{x x}$ versus filling factor for $f=3 \mathrm{GHz}$. (b) Plot of $\operatorname{Im}\left(\sigma_{x x}\right)$ vs $\operatorname{Re}\left(\sigma_{x x}\right)$ for $f=3 \mathrm{GHz}$ (data smoothed by 2 nd order polynominal smoothing for noise reduction). The four curves for different plateau transitions, marked with different symbols, coincide. The data around the not fully resolved plateau $\nu=5$ are omitted. Inset: The same plot for two different frequencies ( $f=3 \mathrm{GHz}$ and $f=5.4 \mathrm{GHz}$ ), each curve including all four transitions in the range $\nu=2-6$. Again the curves collapse to a single function.

$L_{f} \propto f^{-z}$ is the frequency dependent dynamic length. Since $\operatorname{Re}\left(\sigma_{x x}\right)$ depends monotonically on $\delta \nu$ we can invert $\operatorname{Re} \sigma_{x x}(y)$ and replace the argument $y=L_{f} / \xi(\delta \nu)$ in $\operatorname{Im} \sigma_{x x}(y)$ with some function $g^{-1}\left(\operatorname{Re} \sigma_{x x}\right)$. Doing this we expect an explicit, transition, and frequency independent function $\operatorname{Im}\left(\sigma_{x x}\right)=\tilde{g}\left[\operatorname{Re}\left(\sigma_{x x}\right)\right]$. In Fig. 2b, where $\operatorname{Im}\left(\sigma_{x x}\right)$ vs $\operatorname{Re}\left(\sigma_{x x}\right)$ at $f=3 \mathrm{GHz}$ is shown for different transitions, such a universal scaling is indeed observed. We find a similar agreement for all frequencies $f \geq 2 \mathrm{GHz}$. As an example the comparison of the filling factor range $\nu=2-6$ is shown for frequencies of $f=3 \mathrm{GHz}$ and $f=5.4 \mathrm{GHz}$ in the inset of Fig. $2 \mathrm{~b}$. Although the transition width $\Delta \nu$ for the two frequencies, defined as full width at half maximum of the conductivity peaks, differs by a factor of 1.5 the shape in this kind of plotting of $\operatorname{Im}\left(\sigma_{x x}\right)$ versus $\operatorname{Re}\left(\sigma_{x x}\right)$ agrees. This insensitivity on filling factor and frequency confirms the scaling behavior of the sample.

Let us now turn to the second central topic of this Letter, namely a direct experimental access to the localization length $\xi$. To test for conventional scaling behavior we have investigated the temperature and voltage dependence of the plateau transition width $\Delta \nu$. Both follow power laws $\Delta \nu \propto T^{\kappa}$ and $\Delta \nu \propto V^{a}$ with exponents $\kappa \approx 0.43$ and $a \approx 0.22$ as found in most previous experiments [1]. The frequency dependence of $\Delta \nu$ is also found to be in agreement with scaling; for details, see [7].

A similar analysis of $\Delta \nu$ as a function of frequency has already been performed by Engel et al. [10] and Balaban et al. [11] with contradicting results concerning the validity of scaling. However, it is important to stress that although the plateau transition is a powerful tool for the test of scaling, it is only an indirect approach to the localization length. In particular, the failure of transition width scaling found in the data by Balaban et al. [11] does not entail inevitably a failure of universal localization length scaling.

Using Eq. (1) our high frequency measurements allow a direct evaluation of the localization length, provided that $\sigma_{x x}(f) \gg \sigma_{x x}(0)$ for a fixed filling factor. This is equivalent to $\xi \ll L_{T}$, where the temperature length $L_{T}$ is commonly interpreted as inelastic scattering length. Examples for linear fits of our data to Eq. (1) are shown in Fig. 3a.
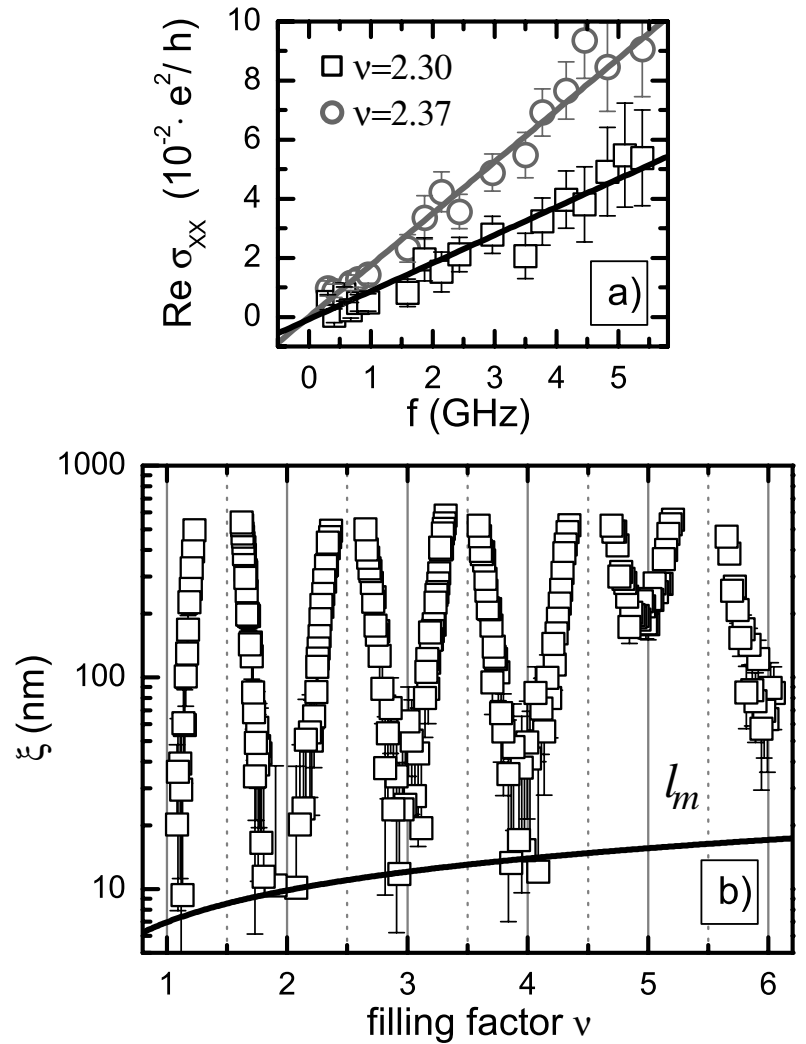

FIG. 3. (a) Linear fit of the frequency dependence for two different filling factors. (b) Localization length $\xi$ as a function of filling factor. The error bars correspond to the uncertainty of the linear fits. Around half-integer filling factors $\sigma_{x x}(f) \gg$ $\sigma_{x x}(0)$ is not fulfilled and thus the determination of $\xi$ is not possible. The solid line denotes the filling factor dependence of the magnetic length $l_{m}$. 


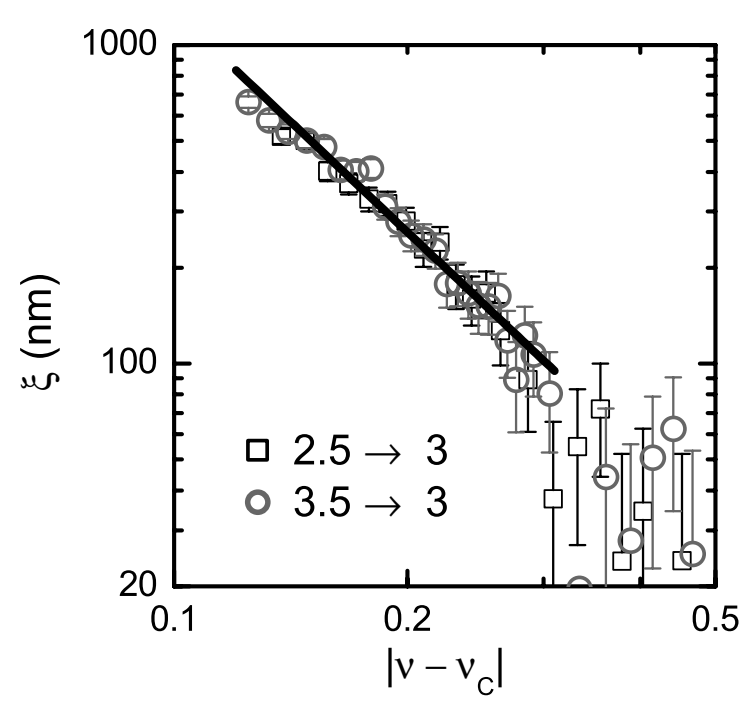

FIG. 4. Localization length $\xi$ as a function of the filling factor difference $\delta \nu=\left|\nu-\nu_{c}\right|$ to the nearest critical point $\nu_{c}$ for both sides of the $\nu=3$ plateau. The straight line corresponds to the expected power law with known universal exponent $\gamma=2.3$ and the prefactor chosen to agree with the data.

Using Eq. (1) with $\epsilon \approx 12$ for GaAs the localization length $\xi$ can be directly extracted from the slope of these linear fits. The resulting dependence of $\xi$ on $\nu$ is plotted in Fig. 3b. In the same graph the filling factor dependence of the magnetic length $l_{m}=\sqrt{\hbar / e B}$ is drawn. Though the error bars are rather large compared to this length the plot shows qualitatively, that the magnetic length, which is the shortest possible length scale of the electron system in a high magnetic field, sets the order of magnitude for the localization length in the middle of the quantum Hall plateaus. The plateaus for $\nu \geq 5$ show a larger localization length because the energy gaps are no longer fully resolved at low magnetic fields.

With this direct measurement of the localization length it is possible to test the scope of the power law $\xi \propto$ $\left|\nu-\nu_{c}\right|^{-\gamma}$ when approaching the QHE plateau. This is shown in Fig. 4 for both sides of the $\nu=3$ plateau. We have restricted the comparison of the experimental data to the expected scaling behavior to a range $0.13 \leq \delta \nu \leq 0.3$. For $\delta \nu \geqslant 0.13$ the sample is well within the variable range hopping regime and an analysis following [2] can be performed. For $\delta \nu \lesssim 0.3$ the proportionality between $\delta \nu=\nu-\nu_{c}$ and the energetic distance to the critical point $\delta E=E-E_{c}$ is valid.

Data and power law with $\gamma=2.3$ agree well over the complete range. The same agreement is found for other plateaus except for filling factors slightly above even integers, where the topmost Landau band is nearly empty. On first glimpse this agreement might be rather astonishing because of the rather large distance from the critical point. However, numerical calculations of Huckestein et al. [14] also show a power law $\xi \propto\left|E-E_{c}\right|^{-\gamma}$ down to a localization length of a few magnetic lengths $l_{m}$.

In conclusion, we measured the frequency dependence of the longitudinal conductivity $\sigma_{x x}$ for frequencies up to $6 \mathrm{GHz}$. We were able to access both the real part and the imaginary part of $\sigma_{x x}$ which allowed us to perform a new test of scaling behavior. Using the theory of Polyakov and Shklovskii on variable-range hopping in the QHE we were able to deduce the localization length. Its filling factor dependence is consistent with universal scaling behavior far into the QHE plateau.

We thank Y. Avishai, F. Evers, B. Huckestein, B. Kramer, F. Kuchar, J. Melcher, D. G. Polyakov, and L. Schweitzer for useful discussions. The sample was grown by K. Pierz at the Physikalisch-Technische Bundesanstalt in Braunschweig and patterned by A. Nauen.

*Electronic address: hohls@nano.uni-hannover.de

[1] Recent reviews: B. Huckestein, Rev. Mod. Phys. 67, 357 (1995); S. L. Sondhi, S. M. Girvin, J. P. Carini, and D. Shahar, Rev. Mod. Phys. 69, 315 (1997).

[2] D. G. Polyakov and B.I. Shklovskii, Phys. Rev. B 48, 11167 (1993); Phys. Rev. Lett. 70, 3796 (1993).

[3] G. Ebert, K. von Klitzing, C. Probst, E. Schuberth, K. Ploog, and G. Weimann, Solid State Commun. 45, 625 (1983); A. Briggs, Y. Guldner, J.P. Vieren, M. Voos, J. P. Hirtz, and M. Razeghi, Phys. Rev. B 27, 6549 (1983).

[4] S. Koch, R. J. Haug, K. v. Klitzing, and K. Ploog, Semicond. Sci. Technol. 10, 209 (1995); M. Furlan, Phys. Rev. B 57, 14818 (1998).

[5] S. Koch, R. J. Haug, K. v. Klitzing, and K. Ploog, Phys. Rev. Lett. 67, 883 (1991).

[6] F. Kuchar, R. Meisels, K. Dybko, and B. Kramer, Europhys. Lett. 49, 480 (2000).

[7] F. Hohls, U. Zeitler, R. J. Haug, and K. Pierz, cond-mat/ 0010417; Physica (Amsterdam) 298B, 88 (2001).

[8] E.g., M. P. A. Fisher, G. Grinstein, and S. M. Girvin, Phys. Rev. Lett. 64, 587 (1990); Y. Huo, R. E. Hetzel, and R. N. Bhatt, Phys. Rev. Lett. 70, 481 (1993).

[9] I. M. Ruzin, N. R. Cooper, and B. I. Halperin, Phys. Rev. B 53, 1558 (1996).

[10] L. W. Engel, D. Shahar, C. Kurdak, and D. C. Tsui, Phys. Rev. Lett. 71, 2638 (1993).

[11] N. Q. Balaban, U. Meirav, and I. Bar-Joseph, Phys. Rev. Lett. 81, 4967 (1998).

[12] A. L. Efros, JETP 62, 1057 (1985).

[13] A. M. M. Pruisken, Phys. Rev. Lett. 61, 1297 (1988).

[14] B. Huckestein and B. Kramer, Phys. Rev. Lett. 64, 1437 (1990). 